\title{
New perspective on atrial fibrillation: A theoretical assumption
}

\author{
Vladimir Tilman* \\ Tne Sheba Medical Center, Rehabilitation Hospital, Ramat Gan, Israel
}

\begin{abstract}
Atrial fibrillation (AF) is the most common cardiac arrhythmia in clinical practice. Many studies have investigated the cause for the development of AF, however, the question remains unanswered. Recently the hypothesis was proposed that atrial fibrillation is a protective physiological mechanism, based on the termination of atrial mechanical systole. This reduces the pressure in the system of the pulmonary veins and alveolar capillaries in pathological situations, and thus reduces the likelihood for development of pulmonary congestion and edema. This hypothesis explains the relationship between left ventrical (LV) dysfunction and AF, helps to build algorithms of relationships between $\mathrm{AF}$ and $\mathrm{LV}$ dysfunction, and with various diseases and conditions, suggests a new approach to the location of the source of $\mathrm{AF}$ and shows a protective function of the atriums in the development of LV dysfunction. The hypothesis is well correlated with the known facts and phenomenons associated with AF.

It is known that atrial fibrillation (AF) is the most common arrhythmia in clinical practice and is most often associated with LV dysfunction / heart failure(HF). It is called the epidemic of the century. An arrhythmia that is particulary common in the elderly, AF is growing in prevalence with the ageing of the population. In addition to age, there are many types of cardiac and medical conditions that are also closely linked to AF. These include hypertention, coronary artery disease, heart failure, valvular heart diseases, hyperthyroidism and so on. Many studies on the subject: "AF - a cause or consequence?" have been carried out, however the question remains unanswered [1].
\end{abstract}

\section{The first question - Why does AF develop?}

In the years 2015-2016 the new hypothesis [2] was proposed, which assumes that $\mathrm{AF}$ is a physiological protective mechanism in the cases of LV dysfunction/heart failure and it answers many questions related to the AF. The reasoning of the hypothesis is as follow. The end diastolic pressure(EDP) is the algebraic sum of the left ventricle diastolic pressure(LVdp) and left atrial systolic pressure(atrial kick pressure, $\mathrm{AKp}$ ). EDP=LVdp+AKP. Normal range of EDP is 8-12 mm Hg, AKp is $2-6 \mathrm{~mm} \mathrm{Hg}$. At the end of the diastole when the mitral valve(MV) is still open, EDP immediately transmitted and equalized (according to hydrodynamic law) throughout the communicating system of the $\mathrm{LV}, \mathrm{LA}$, pulmonary veins(PV), and alveolar capillaries(AC), as there are no obstacles to the spread of the EDP wave. EDP is thus equal to pulmonary wedge pressure(PWP) with a normal range of 8-12 $\mathrm{mm} \mathrm{Hg}$.

In the conditions of LV dysfunction(HF varying degrees), EDP increases due to failure of the LV(i.e. LV diastolic pressure component increases). $\uparrow \mathrm{EDP}=\uparrow \mathrm{LVdp}+\mathrm{AKp}$. This increased EDP at the end of diastole wth opened MV transmits to the entire communicating system of the LA-PV-AC and with the increased PWP, but with the threat of exit of fluid through the alveolar-capillary membrane. Pulmonary edema is usually present when PWP increases to $20 \mathrm{~mm} \mathrm{Hg}$.

It is logical to assume that a physiological protective mechanism would exist to prevent sustained increased pressure in the system described. But to reduce the LV diastolic pressure( $\mathrm{LVdp}$ ) is impossible because it is determined by the pathological condition of the $\mathrm{LV}$ myocardium and is not subject to rapid change. However, it is possible to reduce the total EDP (and to reduce pressure in the entire system!) by stopping the mechanical systole of left atrium, thus, excluding the LA pressure component from the total EDP. EDP $=$ LVdp + AKp.
This is accomplished by AF which stops the mechanical systole of left atrium.The right atrium is also involved in the AF. Termination of RA systole leads to decrease EDP of right ventricle (RV) and to decrease of systemic venous inflow to the heart, resulting in a reduction of preload. It is known that reduction of preload is one of the first important urgent treatments of pulmonary edema. Thus, AF leads to a synergy in the hydrodynamic results, due to the termination of a mechanical systole in both the LA and RA.

Many facts confirm that the termination of mechanical systole of both atriums by the development AF in the cases of LV dysfunction/ HF creates optimal hydrodynamic conditions in the heart when the pressure in the communicating system of LA-PV-AC increases, and therefore, it appears that the heart "NEEDS" AF:

a/ Several clinical trials (AFFIRM, RACE, STAF, AF-CHF etc.) have failed to demonstrate the clinical advantage of sinus rhythm over optimal rate-control. The rhythm-control provided no benefit and actually showed a trend toward harm in the conditions of LV dysfunction/HF compared with rate-control strategy [3-5].

b/ In many cases, after successful treatment of AF (by medications, cardioversion, or ablation) there is a spontaneous return of AF. This tendency of AF to reccur is consistent with the above proposed

Correspondence to: Vladimir Tilman, Tne Sheba Medical Center, Rehabilitation Hospital, Ramat Gan, Eilat st.33, Apt 4, Holon, Israel; Tel: 972-0547345525; E-mail: water5945@gmail.com

Received: August 01, 2016; Accepted: August 24, 2016; Published: August 29, 2016 
hypothesis that the heart in the conditions of LV dysfunction "needs" $\mathrm{AF}$, which gives him more optimal hydrodynamic benefit $[6,7]$.

c/ The occurrence of pulmonary edema after successful return of AF to sinus rhythm is not uncommon in clinical practice cases. This phenomenon is explained by the fact that the hydrodynamics with $\mathrm{AF}$ is preferable for the heart in the conditions of LV dysfunction [8].

d/ The development of pulmonary edema in cases where the pacemaker was implanted for AF can be explained in the same way(so-called pacemaker syndrome). In these cases, there is not only desynchronization of atriums and ventricles, but also a pressure increase in the system of LA-PV-AC as a result of imposing atrial regular rhythm instead of the former AF [9].

Thus, the assuption that $\mathrm{AF}$ is a physiological protective mechanism in the conditions of LV dysfunction/HF explains well all the facts and phenomena associated with the AF and allows us to build the algorithms of their relationships in various diseases [10]. It is possible that $\mathrm{AF}$ ia a genetically programmed mechanism for primary protection from increasing pressure in the LA-PV-AC system due to pathological conditions. Aberrant triggering of this mechanism at the genetic level, perhaps due to mutation, may explain the development of AF with no apparent organic pathology - Lone AF.

$\mathrm{AF}$ is not without drawbacks, like many other well-known protective and compensatory mechanisms; the most prominent of which is its tendency towards thrombosis in the LA. Rapid rate of AF ( a function of the AV node) can have the potential to exacerbate HF.

\section{The second important question - Where is the source of Af?}

There are several theories as to the development of AF, but no one answeres the question where is its source. Localisation of an anatomical or histological substrate as a source of AF has still not been found. The good outcomes in the termination of AF by ablation of the pulmonary vein orifices are a strong argument that the patients with AF have a focal (e.g. pacemacer or triggered dischages) mechanism in the region of the pulmonary veins, and it is likely that ablation only "cuts" the spread of AF. This is consistent with the proposed hypothesis because the estimated source of AF is in a strategically important localization in the LA-PV-AC system, where increased pressure can lead to complications (pulmonary cogestion or edema). This increase of pressure in the entire system can activate the mechanism of AF localised in the pulmonary veins. Therefore, the purpose of the development of $\mathrm{AF}$ is to reduce the pressure in the system. It also possible that the source of AF have functions of baroreceptors that can "turn on" AF when there is risk of increasing pressure in the LA-PV-AC system and "turn off" with the disappearance of the danger. In clinical practice, $\mathrm{AF}$ is known to occur in acute pulmonary edema, with spontaneous termination after successfully treating the pulmonary edema without using antiarrhythmic medecines.

Thus, the proposed hypothesis can suggest a scenario for the development of AF. Each individual has a protective physiological mechanism in the pulmonary veins, most likely genetically programmed. When the pressure in the LA-PV-AC system increases to a certain critical value, the AF mechanism is activated. AF terminates mechanical systole of the LA excluding the component of systolic left atrial pressure from the total pressure in the LA-PV-AC system, and of the RA with effect of reducing preload, and thus decreases the threat of the development of pulmonary congestion or edema.
Table 1. Sinus Rhythm and Atrial Fibrillation.

\begin{tabular}{|c|c|}
\hline SINUS RHYTHM & ATRIAL FIBRILLATION \\
\hline $\mathrm{EDP}=\mathrm{DPl}+\mathrm{AKp}$ & $\mathrm{EDP}=\mathrm{DPlv}$ \\
\hline
\end{tabular}

1. In this light, the proposed hypothesis reveals the special role of the left atrium as the protective organ in the conditions of the LV dysfunction. From hydrodynamics, it is known that it is possible to reduce pressure in a system; or directly by decreasing the pressure, or due to an increase of the volume of the system.It is applicable also to the hydrodynamics system of the heart. Three protective mechanisms reducing pressure in the LA-PV-AC system are known.

1/ DIRECT DECREASING PRESSURE in the LA-PV-AC system - the terminating of mechanical systole of the LA at the AF while excluding the component of systolic LA pressure from total EDP (the proposed hypothesis). Thus, AF is the first aid in the conditions of LV dysfunction (Table 1).

EDP - total end dyastolic pressure at the end of LV dyastole,

DPlv - dyastolic pressure of left ventricle at the end of dyastole,

$\mathrm{AKp}$ - systolic left atrial pressure ( atrial kick).

2/ In the case of prolonged overload pressure in the LA-PV-AC system - INCREASING THE VOLUME of the system due to significant dilatation of left atrium, reduces pressure in the system, protecting the patient from the death. In mitral stenosis, where there is prolonged increased pressure in the LA-PV-AC system, a significant enlargement of the LA occurs, sometimes reaching to a gigantic size [11]. On the one hand, it is obvious that the LA, having thin walls, expands under long influence of an elevated pressure in the LA-PV-AC system. On the other hand, this increase in capacity volume of the system would reduce the pressure, and may be the protective mechanism (damper effect) that saves the patient's life. This increase in capacity volume of the system would reduce the pressure similar to dampers used in technical hydrodynamic systems, and may be the compensatory mechanism that save the patient's life. The size of the LA has a direct correlation with $\mathrm{AF}$, underscoring their synergy of action.

3/ HUMORAL protective mechanism - pressure decreases in the LA-PV-AC system due to reduction of volume of liquid - diuretic effect of atrial-natriuretic peptid (ANUP), allocation of which increases in the cases of increasing pressure in the system [12].

\section{Conclusion}

The above proposed hypothesis suggests that AF is a protective physiological mechanism, most likely genetically programmed. The main purpose of $\mathrm{AF}$ is eliminate the mechanical systole of the LA to reduce pressure in the LA-PV-AC system, and of the RA with the effect of reducing preload, that creates more optimal hydrodynamic conditions for the heart in the LV dysfunction / HF and thus decreases the threat of pulmonary congestion or edema. This hydrodynamic approach explains the direct correlation of $\mathrm{AF}$ with all diseases as well as LV dysfunction and age, and with all facts and phenomena associated with AF.

\section{References}

1. Ringborg A, Nieuwlaat R, Lindgren P, Jönsson B, Fidan D, et al. (2008) Costs of atrial fibrillation in five European countries: results from the Euro Heart Survey on atrial fibrillation. Europace 10: 403-411. [Crossref]

2. Tilman V (2016) Atrial fibrillation: In the light of new hypotheses. Med Hypotheses Vol 86: 10-13. 
3. Wyse DG, Waldo AL, DiMarco JP, Domanski MJ, Rosenberg Y, et al. (2002) A comparison of rate control and rhythm control in patients with atrial fibrillation. $N$ Engl J Med 347: 1825-1833. [Crossref]

4. European Heart Rhythm Association, European Association for Cardio-Thoracic Surgery, Camm AJ, et al. (2010) Guidelines for the management of atrial fibrillation: the Task Force for the Management of Atrial Fibrillation of the European Society of Cardiology (ESC). Eur Heart J 31: 2369.

5. Chung MK, Shemanski L, Sherman DG, et al.(2005) Functional status in rate- versus rhythm-control strategies for atrial fibrillation: results of the Atrial Fibrillation FollowUp Investigation of Rhythm Management (AFFIRM) Functional Status Substudy. J Am Coll Cardiol 46: 189.

6. Vikman S, Mäkikallio TH, Yli-Mäyry S, Nurmi M, Airaksinen KE, et al. (2003) Hear rate variability and recurrence of atrial fibrillation after electrical cardioversion. Ann Med 35: 36-42. [Crossref]
7. Alcaraz R, Rieta JJ (2009) Time and frequency recurrence analysis of persistent atrial fibrillation after electrical cardioversion. Physiol Meas 30: 479-489. [Crossref]

8. RM Gowda, D Misra, IA Khan, P Schweitzer (2003) Acute pulmonary edema after successful cardioversion of atrial fibrillation. Am Jther 10: 73-74.

9. Bailey SM, Wilkoff BL (2006) Complications of pacemakers and defibrillators in the elderly. Am J Geriatr Cardiol 15: 102-107. [Crossref]

10. Tilman V (2016) Algorithm of Relationships between Left Ventricle Dysfunction and Atrial Fibrillation. J cardiovasc Dis Diagn 4: 247

11. Tung R, DeSanctis R (2004) Images in clinical medicine. Giant left atrium. $N$ Engl J Med 351: 1437. [Crossref]

12. Saito Y (2010) Roles of atrial natriuretic peptide and its therapeutic use. J Cardiol 56 : 262-270. [Crossref]

Copyright: (C2016 Tilman V. This is an open-access article distributed under the terms of the Creative Commons Attribution License, which permits unrestricted use, distribution, and reproduction in any medium, provided the original author and source are credited. 